\title{
Les Simulies d'Algérie (Diptera : Simuliidae). I. Premières données biogéographiques et écologiques sur les espèces de l'Ouest-algérien
}

J. Gagneur ${ }^{1}$

M. Clergue-Gazeau ${ }^{2}$

Mots clés : Simuliidae, Algérie, faunistique, zoogéographie.

48 stations ont été prospectées sur les oueds du Nord-Ouest de l'Algérie, entre $1145 \mathrm{~m}$ d'altitude et le niveau de la mer: 20 espèces de Simulies ont été récoltées. Un genre, un sous-genre et 7 espèces sont cités pour la première fois en Algérie : une espèce est nouvelle pour l'Afrique du Nord.

Mis à part Simulium (Wilhelmia) pse udequinum, d'origine orientale, la faune appartient à la zone paléarctique (au Sud de la sous-région méditerranéenne) et s'adapte à de nouvelles caractéristiques des cours d'eau (température, salinité...); ainsi, les espèces de plaine de la bordure septentrionale méditerranéenne remontent dans un crénal de moyenne altitude et se retrouvent ici avec les espèces de sources.

Les éléments orophiles sont très peu représentés.

Simulifdae of Algeria (Diptera : Nematocera). I. Biogeographical and ecological data on the species of West Algeria.

Keywords : Simulidae, Algeria, faunistic, zoogeography.

48 sites have been examined in the " oueds * (= wadi) of North-West Algeria between sea-level and a height of 1145 m. 20 species of Simuliidae have been collected. One genus, one sub-genus and seven species are recorded for the first time for Algeria ; one species is a new record for North Africa.

Apart from Simulium (Wilhelmia) pseudequinum which is of eastern origin, the fauna belongs to the palaearctic zone (to the south of the mediter ranean subregion), and is adapted to the new characteristics of the watercourses (temperature, salinity...). Therefore, the species of the plain next to the mediterranean in the north have ascended to a mean altitude and are found together with species occuring at the source.

Orophilic (moutain-loving) elements are very poorly represented.

\section{Introduction}

Jusqu'à ces dernières années, les Simulies n'ont pas fait l'objet de recherches particulières en Algérie comme c'est le cas, depuis deux décennies, sur les territoires tunisien et surtout marocain (BaillyChoumara et al. 1970, Bailly-Choumara \& Beaucournu-Saguez 1978, 1981 ; Giudicelli \& Dakki 1984 ; Boumaiza \& Clergue-Gazeau 1986 ; Bouzidi \& Giudicelli $1987 \ldots$... La plupart des travaux sur ces

1. Université Paul Sabatier, Laboratoire d'Hydrobiologie, UA 695 C.N.R.S., 118, route de Narbonne. 31062 Toulouse Cedex.
Diptères ne fournissent que des données ponctuelles et anciennes (Gauthier 1928, Parrot 1949, Grenier 1953, Vaillant 1955...); seul, Edwards (1923) avait recensé 6 espèces.

Nous exposons ici les résultats obtenus au cours d'une étude hydrobiologique réalisée par l'un de nous (J.G.) sur les réseaux hydrographiques du Nord-Ouest du pays (Wilaya de Tlemcen). Plus de $870 \mathrm{~km}$ de rivières appartenant au bassin de la Tafna et aux bassins côtiers adjacents, ont été prospectés depuis 1981. Tout récemment, d'autres études hydrobiologiques ont débuté dans la partie Est, en grande Kabylie sur l'oued Aïssi (Aït Mouloud 1987,

Article available at http://www.limnology-journal.org or http://dx.doi.org/10.1051/limn/1988024 
Lounaci 1987). Ainsi, par une prospection suivie de cours d'eau à travers le pays, nous pourrons avoir une idée plus précise de la répartition biogéographique des đifférentes espèces, en Algérie et sur l'ensemble du Maghreb.

\section{Région et localités d'étude}

L'oued Tafna prend sa source à $1090 \mathrm{~m}$ dans les monts de Tlemcen (Atlas tellien) et atteint la Méditerranée après un parcours de $170 \mathrm{~km}$ vers le Nord. Cette région, comme la plaine d'Oujda et toute l'Oranie, subit laction at ténuée des vents atlantiques en raison des reliefs du $R$ if marocain et de la Sierra Nevada espagnole, et n'est que peu soumise aux influences sahariennes. Le climat varie, de subhumide en altitude à subaride dans la plaine de Maghnia. La plupart des oueds choisis sont alimentés par des sources et de ce fait, ont un écoulement permanent. Parfois de fortes cfues perturbent les milieux lotiques. Du fait de la nature du substrat et d'une évaporation importante, un taux de salinité élevé est constaté en basse altitude.

L'échantillonnage a été réalisé sur 48 stations : 24 sur le réseau hydrographique de l'oued Tafna, 11 sur celui de son principal affluent l'Isser et 8 sur ceux des oueds côtiers (fig. 1). La liste des localités est donnée dans le Tableau 1, ainsi que les codes figurant sur la carte ef les paramètres utilisés dans ce travail.

\section{La faune}

\subsection{Méthodes de récoltes}

Prélèvements réalisés au filet Surber -surface : $1 / 10 \mathrm{~m}^{2}$; vide de maille: $300 \mu$.. Chaque station regroupe souvent un ensemble d'habitats ; aussi, 3 échantillons ont-ils été prélevés par station. Les larves $(L)$, les nymphes $(N)$ et les exuvies nymphales (E) ont été récoltées dans le benthos. L'échantillonnage dés stades immatures a pu êt re complété par la capture de nombreux imagos mâles (M) ou femelles (F) au filet entomologique, ce qui nous a permis de préciser ou de confirmer la détermination de plusieurs taxa.

A chaque sortie, ont été notées : la température de l'eau, la vitesse du courant, la turbidité et la densité du couvert végétal (Tableau I).
- vitesse du courant (échelle de Berg) $\mathbf{M}:$ moyenne, $\mathbf{L}:$ lente, $\mathbf{L} \mathbf{L}$ : très lente $\simeq \simeq$ eau renouvellée. De faibles débits ont toujours été constatés.

- couvert végétal bordant : $+:$ dense, $>80 \%$; \pm : moyen, $<80 \%$ et $>10 \% ;-:<10 \%$, faible ou inexistant.

— turbidité : CC : eau très limpide; $\mathbf{C}$ : claire; $\simeq \mathrm{C}$ : légèrement trouble; $\mathrm{T}$ : trouble; $\mathrm{TT}:$ très trouble.

Dans la dernière colonne du tableau : A : assèchement temporaire possible de courte durée : $\mathrm{P}$ : pollution nette; $\mathrm{PP}$; pollution aiguë ; $\mathrm{Sa}$; eau salée ; $\mathrm{Sc}$ : source.

Nos prélèvements ont été répartis sur plusieurs mois à différentes saisons, entre 1981 et 1987 .

\subsection{Inventaire faunistique}

* Greniera fabri Doby \& David 1959

* Prosimulium (Prosimulitum) faurei Bernard, Grenier \& Bailly-Choumara 1972 P. (P.) gr. aculeatum Simulium (Eusimulium) velutinum (Santos Abreu 1922) = latinum (Rubzov 1962) n. syn.

* $S$. (E.) angustipes Edwards $1915=$ latizonum (Rubzov 1956) n. syn.

S. (E.) gr. aturetrm

* $S$. (Nevermannia) costatum Friederichs 1920

S. (N.) cf cryophiltam

$S$. (N.) gr. verntum

* S. (N.) ibleum (Rivosecchi 1966) + S. (N) latigonium (Rubzov 1956)

* S. (Obuchovia) marocanum Bouzidi \& Giudicelli 1987

S. (Simulium) intermedium Roubaud 1906

$=$ nitidifrons Edwards 1920 n. syn.

\section{E,F}

N,E,M

$\mathbf{L}$

L, N,E,M,F

L,NE,M,F

$\mathbf{L}, \mathrm{E}$

$\mathbf{L}$

L,E,C

L, F

L,E,C,F.

L.E,M,F

L, N,E,M.F

$L_{i} N, E, M, F$

S. (S.) ornatum Meigen 1818 (complexe

d'especes)

S. (S.) gr. ornatum

S. (S.) gr. reptans

* S. (S.) gaudi Grenier \& Faure 1956

S. (Tetisimulium) bezzii (Corti 1916)

S. (Wilhelmia) pseudequinum Seguy 1921

S. (W.) sergenii Edwards 1923

$\mathbf{L}, \mathbf{N}, \mathbf{E}, \mathbf{M}, \mathbf{F}$

E

L.

$\mathbf{L}, \mathbf{N}, \mathbf{E}, \mathbf{M}, \mathbf{F}$

$L, N, E, M$

L,N,E,M,F

L,N,E,M,F

* espèce non encore signalée d'Algérie,

+ espèce nouvelle pour l'Afrique du Nord.

Cette liste suit l'ordre de classification de Crosskey (1981) et tient compte des nouveaux synonymes de sa récente publication : "Checklist of the world blackflies »(1987). Dans cette dernière, le groupe ornatum (ancien sous-genre Odagmia) est 


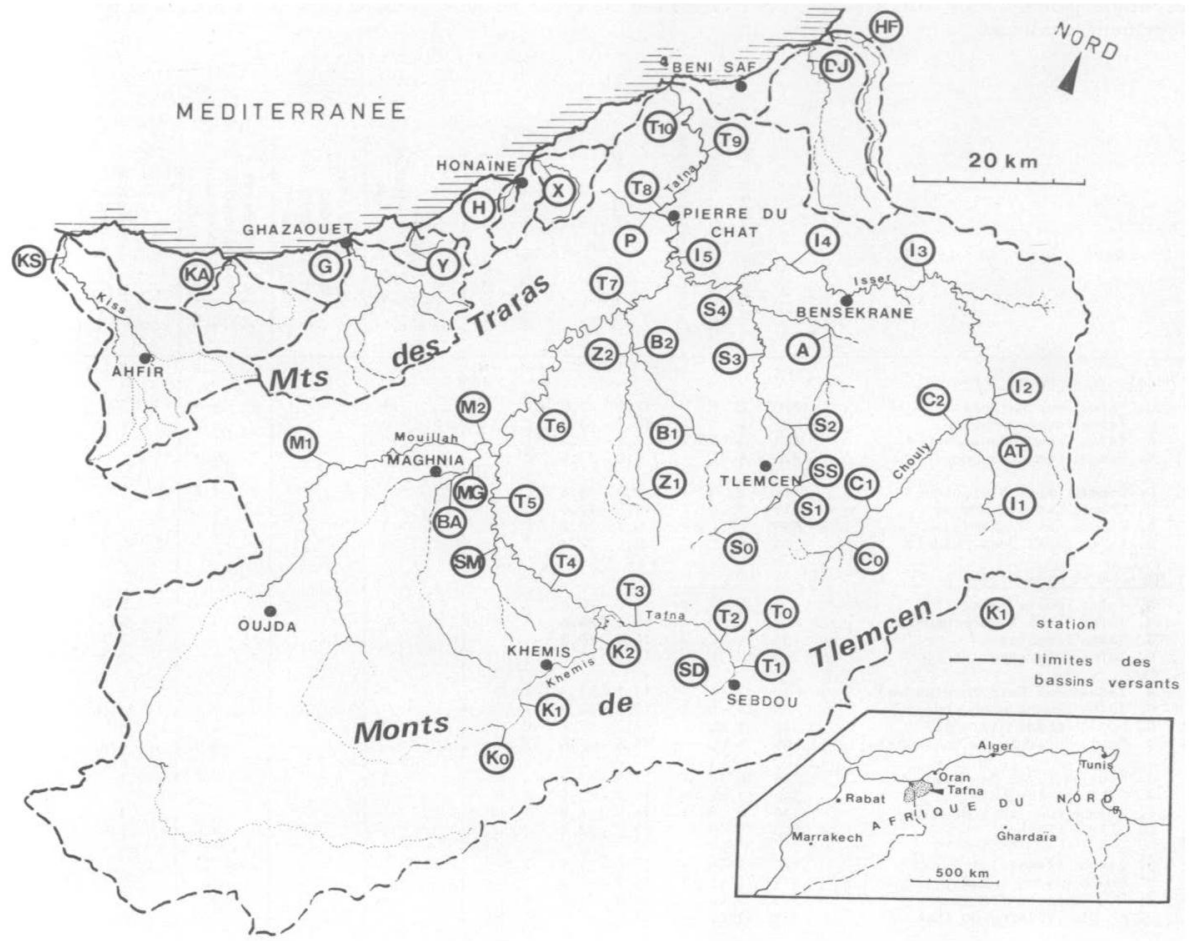

Fig. 1: Carte de la région étudiée. Réseau hydrographique de l'oued Tafna et des oueds côtiers, avec les 48 stations.

placé dans le sous-genre Simulium et le groupe aculeatum (ancien sous-gen re Urosimulium) dans le sous-genre Prosimulium.

\subsection{Biogéographie et répartition des espèces}

Jusqu'à ces dernières années, 7 taxons étaient recensés en Algérie : $S$. (E.) gr. aureum $(=$ velutinum, S. (S.) intermedium, S. (S.) omatum, S. (N.) ruficome, $S$. (T.) bezzii, S. (W.) pseudequinum et $S$. (W.) sergenti. Tout récemment, en grande Kabylie, l'étude hydrobiologique et l'oued Aïssi a donné 4 espèces non encore signalées: $S$. (N.) cryophilum, $S$. (S.) variegatum, $S$. (S.) argenteostriatum et $S$. (S.) gr. monticola; ce qui portait à 11 le nombre d'éléments inventoriés.

Dans notre étude, 20 espèces ont été recensées ; 7 sont, à notre connaissance, nouvelles pour l'Algérie : $P$. ( $P$.) faurei, $P$. (P.) gr. aculeatum, $S$. (E.) angustipes, $S$. (N.) ibleum, $S$. (N.) latigonium, S. (Ob.) marocanum et $S$. (S.) gaudi, une l'est pour l'Afrique du Nord: $S$. (N.) latigonium, et 4 ne sont identifiées qu'au groupe. 
Tableau I. - Liste des 48 stations de récolte, avec leur principaux paramètres physiques et leur peuplement : richesse spécifique. (abréviations dans le texte). Les espèces ont été réparties en 4 groupes de 4 afin d'alléger et de lire plus facilement le tableau.

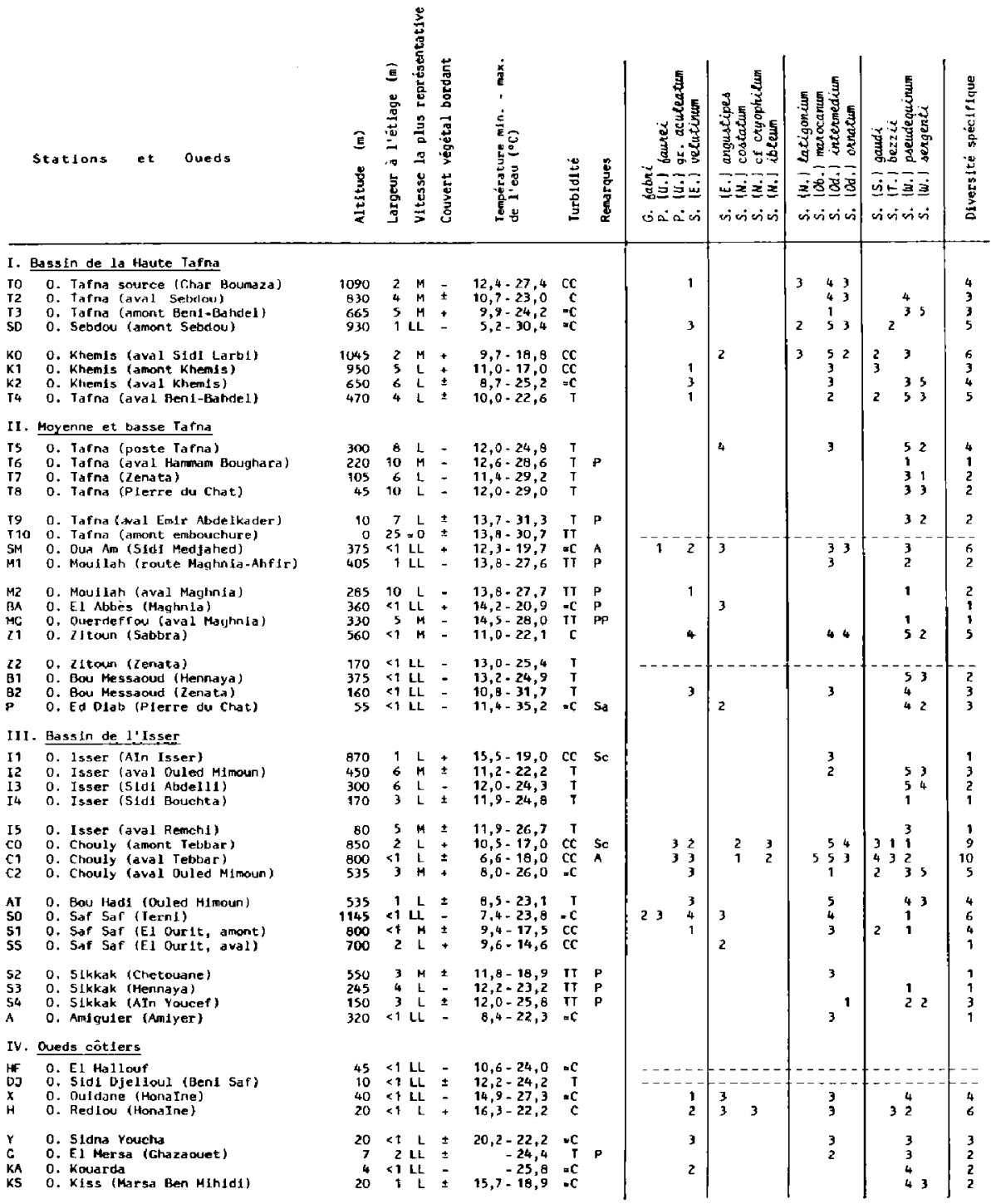


5 classes rendent compte de l'abondance relative des 16 taxons identifiés à l'espèce ou affines. (Tableau I).

classe $1: 1$ individu, espece rare ou accidentelle ; classe 2: 2-5 ind., espèce peu abondante;

classe $3: 6-30$ ind., espèce assez abondante ;

classe 4 : 31-100 ind., espèce abondante ;

classe $5:>100$ ind., espèce très abondante.

Le calcul de l'abondance est basé sur des effectif's de larves et de nymphes cumulés et prélevés au filet Surber.

La fréquence (Tableau II) est chiffrée par le coefficient :

$\mathrm{Cf}=\underline{\text { Nombre de st. où l'espèce est récoltée }} \times 100$ Nombre total de stations

Les Simulies peuvent ainsi être classées en :

- esp. abondantes et très fréquentes (= dominantes) : S. (W.) pseudequinum, S. (S.) intermedium; esp. abondantes et fréquentes : $S$. (E.) velutinum, $S$. (W.) sergenti; esp. assez fréquentes et assez abondantes : S. (E.) angustipes, S. (S.) ornatum, S. (S.) gaudi ; esp. localisées : S. (Ob.) marocanum; esp. peu fréquentes et pez abondantes : S. (T.) bezzii, S. (N.) ibleum, S. (N.) cf. cryophilum, P. (P.) faurei, G. fabri,...

\subsection{Espèces caractéristiques}

\section{Greniera fabri}

Décrite de France occidentale (Bretagne : Doby \& David 1959), l'espèce est réputée assez rare; jusqu'à ces dernières années, sa répartition s'est en effet limitée à quelques gîtes en France occidentale et méridionale, en Sardaigne et sur la côte occidentale de l'Italie (Latium et Calabre): Rivosecchi (1978) parle d'une répartition occidentale méditerranéenne. Cette opinion est renforcée désormais par la découverte de l'espèce-type en Algérie (st. SO). Très récemment, à l'occasion d'une description d'espèce nouvelle du même genre en Israël, BeaucournuSaguez \& Braverman (1987) signalent sa présence au Maroc; ces deux nouvelles stations maghrébines: Marac, où l'on ne connait pas encore le lieu de capture, et région ouest de l'Algérie, étendent son aire de répartition à la bordure sud de la Méditerranée.
Tableau II. - Fréquence et classe drabondance la plus représentative des espèces rencontrées.

\begin{tabular}{|c|c|c|c|}
\hline & 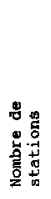 & 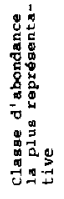 & 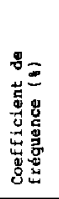 \\
\hline G. fabri & 1 & 2 & 2,1 \\
\hline P. I p.J faurei & 2 & 3 & 4.2 \\
\hline P. (P.) gr. aculeatum & 2 & 3 & 4.2 \\
\hline S. (E.) velutinum & 19 & 3 & 39.6 \\
\hline S. (E.) angustipes & 9 & 3 & 18,8 \\
\hline s. (N.) costatum & 2 & 2 & 4,2 \\
\hline 5. (N.) of aryophilum & 1 & 3 & 2,1 \\
\hline s. (N.) ibleun & 2 & 3 & 4,2 \\
\hline S. (N.) latigonium & 3 & 3 & 6.3 \\
\hline s. (ob.) marocanum & 1 & 5 & 2,1 \\
\hline S. (S.) intermediun & 27 & 3 & 56.3 \\
\hline s. Is ; ornatum & 9 & 3 & 18,6 \\
\hline S. (S.) gaudi & 7 & 2 & 14,6 \\
\hline S. (T. $\}$ bezzii & 4 & 3 & 8,3 \\
\hline s. (H.) pseudequinum & 35 & 3 & 73,0 \\
\hline s. (h.) sergenti & 16 & 3 & 33,3 \\
\hline
\end{tabular}

Son écologie a été précisée par BeaucournuSaguez et al. (1969) : présente dans de petits ruisseaux ou rivières de plaine où la végétation est nécessaire en raison de la biologie de la nymphe ; sans cocon, elle vit en effet dans les herbes, accrochè à des fils de soies. En Algérie, G. fabri a été capturée également dans un ruisseau dont les caractéristiques mésologiques correspondent sensiblement à celles ind iquées pour les gîtes de Bretagne. Comme dans ceux-ci, l'espèce ne semble y être ni fréquente, ni abondante. La présence d'exuvies nymphales et de femelles au mois de mai coïncide avec le déroulement du cycle en France.

\section{Prosimulium (Prosimulium) faurei}

Ce taxon est décrit du Maroc (Bernard et al. 1972) près de Meknès. Jusqu'à présent, il n'avait pas encore été signalé en dehors de cette région du Moyen-Atlas.

En Algérie, P. (P.) faturei colonise une source (SO) et un émissaire de source (SM).

\section{P. (P.) gr. aculeatum}

Cette espèce est proche de P.(P.) jucci Contini, principalement par l'aspect du processus tp de la 
nnandibule chez la larve, bombé et pourvu de 7 à 11 petites dents et par quelques autres détails morphologiques; mais, elle possède une différence très importante dans le dispositif des dents du bord supérieur du submentum. La dent trifide des taxa connus est située soit légèrement au-dessus, soit au niveau des dents intermediaires les plus hautes ; en outre, les dents intermédiaires sont bien plus petites que les latérales, l'ensemble formant un " $V$ " plus ou moins ouvert (fig. $2 \mathrm{C}$ et D). Contrairement aux précédents, le submentum observé ici possède des dents intermédiaires et latérales situées sur une mème ligne horizontale et la dent centrale se situe très en-dessous de cette ligne (fig. 2E).

Aux mois de décembre et février, les larves se trouvaient dans une source (CO) et son émissaire $(\mathrm{Cl})$ à écoulement lent, eau très claire et incrustante; ce biotope semble assez différent de celui de l'espècetype, eury therme, qui se complait dans les milieux eutrophes, algues vertes ou débris végétaux (Sardaigne: Rivosecchi 1978; Tunisie: Boumaiza \& Clergue-Gazeau 1986).

Récemment, Gonzalèz-Peña et al. (1986) ont mentionne une forme proche de $P$. $(P$.) aculeatum, capturée dans le rio Yeguas au Sud de l'Espagne; celleci établit la jonction entre les populations insulaires et maghrébines du groupe.

\section{Le groupe aureum}

Il est représenté par trois éléments : deux espèces bien connues des régions méditerranéennes, $S$. (E.) velutinum et $S$. (E.) angustipes et un troisième (st. Cl) à filaments branchiaux de la paire supérieure chez la nymphe- de fort diamètre, la paire inférieure restant mince et pratiquement sessile.

Simulium (Nevermannia) cf. cryophilum

Par de nombreux caractères taxonomiques, cette forme pourrait être assimilée à $S$. (N.) cryophilum ; cependant, elle présente quelques différences de détails avec elle, la plus importante étant le processus médian antérieur du cocon (de type vernum) remplacé ici par une sorte de bourrelet (fig. 2B). L'absence d'imagos mâles ne nous permet pas, pour l'instant de préciser sa position taxonomique.

Larves et nymphes ont été recueillies dans une seule station $(\mathrm{H})$ : un ruisseau côtier de basse altitude, avec eau limpide et une vitesse de courant lente, d'où une température de l'eau assez élevée. Ce biotope n'est pas, à proprement parler, celui de l'espèce-type, que l'on capture dans des cours d'eau à courant vif et bien oxygénés (Tunisie: BaillyChoumara et al. 1970 ; Boumaiza \& Clergue-Gazeau 1986).

Espèces associées: le groupe aureum, S.(S.) intermcdium, S.(S.) gaudi et S. (W.) pseudequinum.

\section{Simulium (Nevermannia) latigonium}

Non encore signalé en Afrique du Nord, ce taxon a une répartition européenne et s'étend du Danemark à l'Espagne; il est plutót rare et ses populations ne sont jamais abondantes. Dans ces pays, son biotope est caractérisé par un courant lent, une richesse en matjères organiques et des températures estivales élevées.

Situées dans la haute Tafna, les 3 stations (TO, SD. KO) où nous l'avons récol té présentent sensiblemęnt le même biotope : ruisseaux à courant lent (KO) ou modéré, végétation immergée (Renoncules et Potamots) et température estivale particulièrement éltevée (TO : $27^{\circ} 4$; SD : $30^{\circ}$ ) ; l'eau y coule très claire et provient de sources de moyenne altitude (900-1100 m). La haute teneur en calcium constatéc caractérise également plusieurs stations françaises de l'espece (Vinçon \& Clergue-Gazeau 1987).

Especes associées: $S$. (S.) intermeditum et $S$. (S.) omatum.

\section{Simulium (Nevermannia) ibleum}

Déjà inventorié récemment en Tunisie (Boumaiză \& Clergue-Gazeau 1986), cet te deuxième capture du taxon pour l'Afrique du Nord permet d'étendre sa répartition à l'Ouest du pourtour méditerranéen : il était connu auparavent de Sicile (localité-type), du Liban et de Cyrénaique.

Comme la plupart des éléments du groupe angustitarse, S.(N.) ibleum est présent dans une source (CO) et son émissaire ( $\mathrm{Cl})$, à température minimale froide $\left(6^{\circ}\right)$ et concrétions; ces 2 nouveaux gîtes confirment l'appartenance de l'espece au crénal; une faible population larvaire est présente au mois de mars (avec imagos en mai), comme celles du Liban et de Tunisie.

\section{Simulium (Obuchovia) marocanum}

L'espèce vient d'être décrite au Maroc par Bouzidi \& Giudicelli (1987); du point de vue taxonomique, elle est très proche de S. $(O b)$ galloprovinciale, du Sud de la France. Nous rattachons nos exemplaires à $S$. (Ob.) marocanum, les mâles (ex. nympha) présentant une plaque ventrale large. Le sclérite médian nous semble moins étroit dans sa partie médiane qu'aux extrémités et de ce fait, le parallélisme des 


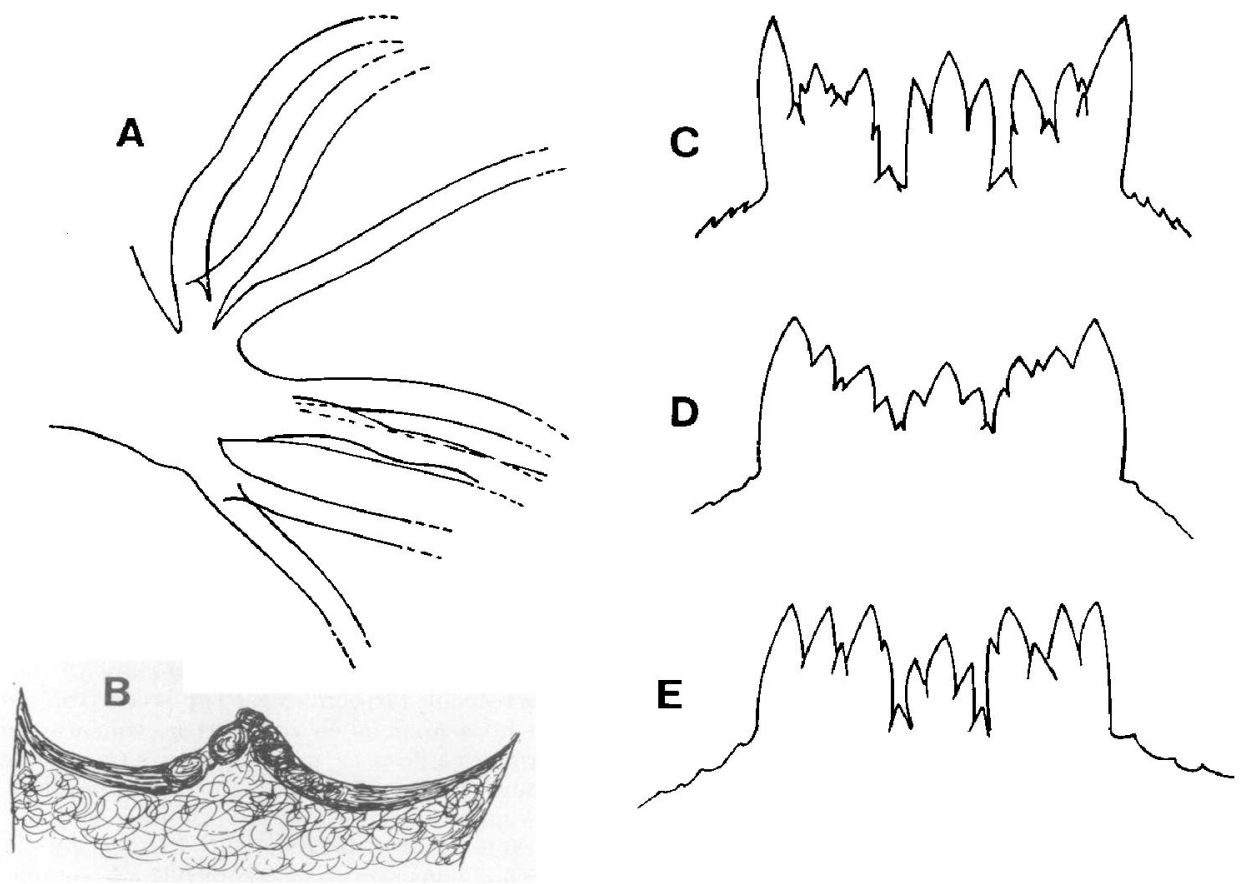

Fig. 2. - Particularités morphologiques de plusieurs espèces.

A : filaments branchiaux de S.fS.) gr. ornatum (st. Cl.); B : extrémité antérieure du cocon de $S$. (N.) cf. cryophilum ; ext rémité antérieure du submentum de: C:P. (P.) faurei; D : P. (P.) juccii : E : P. (P.) gr. aculeatum.

bords n'apparaît pas toujours. Chez la nymphe, le tégument céphalique est simplement bosselé par endraits et ne présente pas de plis profonds.

Larves et nymphes vivent dans un émissaire de source $(\mathrm{Cl})$ où l'eau est incrustante et cimente les galets du fond ; végétation bordante et minima de température peu élevés $\left(6^{\circ} 4\right)$. Les larves et nymphes ont été capturées dans la partie la plus dénivellée du cours d'eau. La présence de nombreuses nymphes mûres au mois de mai précède l'envol des imagos avant l'élévation de tempéra ture estivale et l'assèchement de l'oued. Pas d'espèces associées.
Simulium (Simulium) gr. ornatum

Nous avons séparé le sous-genre en trois :

$1^{\text {e }}-$ S. (S.) intermeditum s. str.; $2^{\text {e }}$ - S. (S.) omatum s. l. (complexe duquel est exclue l'espèce précédente) ; $3^{e}$ - une forme différente appartenant vraisemblablement au gr. omatum par la morphologie de l'exuvie nymphale et l'orientation des filaments branchiaux. Les troncs communs sont élargis à la base et les filaments presque sessiles, avec élargissement de l'angle séparant les filaments de la deuxième paire (fig. 2A). Cet élément vit en association étroite avec S. (S.) internedium (Cl). 


\section{a) S. (S.) intermedium}

Grâce à la dissection des imagos mâles et à l'observation externe des femelles, il nous a été possible de confirmer avec précision les stations ou l'espèce est présente. Avec 27 st., c'est la Simulie la plus fréquẹte après $S$. (W.) pseudequinum et l'élément dominant du groupe. De vaste répartition, recouvrant l'Europe et le pourtour méditerranéen, elle est très commune ici comme au Maroc, à toutes altitudes et dans des biotopes très variés. Vers l'Est du Maghreb, par contre, sa présence se limite à un petit nombre de stations au Nord-Ouest de la Tunisie.

b) S. (S.) ornatum. s.l.

Nous avons regroupé des formes possédant les génitalia mâles à styles larges et des troncs communs nets aux filaments branchiaux de la nymphe; il semblerait qu'il existe deux aspects morphologiques de ces derniers, les 2 trones communs des deux paires de filaments supérieurs étant reliés ou non par un court pédicule. Parmi ces formes, on trouve des cocons à texture plus ou moins serrée, ce qui ajoute à l'incertitude quant au polymorphisme d'une ou de plusieurs formes. (Rappelons à cet égard la forme libanaise de l'espèce $S$. (S.) ornatum, avec cocon à texture lâche). Le problème de ces formes ne peut être résolu qu'avec l'étude des chromosomes polytènes de la larve.

Avec 9 st., S. (S.) omatum s.l. est bien moins répandue que l'espèce précédente; toutes deux sont étroitement associées dans les sources et leurs émissaires (CO et $\mathrm{Cl}$...).

Autre espèce associée en dehors des sources: $S$. (W.) pseudequinum.

\section{Simulium (Simulium) gaudi}

Décrit au Maroc (Grenier \& Faure 1956) et nouveau pour l'Algérie, ce taxon est répandu en Europe occidentale; d'après Crosskey (1987), ce pourrait être un synonyme de $S$. (S.) xanthinum Edw. 1933. Il n'est pas très fréquent, sa présence étant liée à un type de milieu bien particulier : ruisseau calcaire émissaire de source (avec tufs).

En Algérie, S.(S.) gaudi colonise le même type de biotope : elle est présente dans 7 ruisselets de source et particulièrement abondante dans une source et son émissaire ( $\mathrm{CO}$ et $\mathrm{Cl}$ ) avec des eaux incrustantes.

\section{Simulium (Wilhelmia) sergenti}

Connue depuis longtemps d'Algérie-Edwards l'a décrite en 1923 près d'Alger et de Biskra-, l'espèce esı . ndue dans tout le Maghreb et se retrouve avec moins de fréquence, à l'extrême Sud de l'Europe occidentale (Portugal, Espagne, BeaucournuSaguez 1972, 1975). Elle colonise des milieux variés de basse altitude (16 st.); son abondance parait maximale dans les rivières de piémont à températures élevées -K2, I2, I3 et C2-, aux débits élevés et assez stables relativement à ceux de la région, mais dont la turbidité des eaux et la conductivité montrent une transition entre les parties supérjeures et inférieures des oueds.

Espèce associée : la plupart du temps avec $S$. (W.) pseudequinum.

\section{Discussion et conclusion}

Vingt espèces sont actuellement recensées sur le territoire algérien : 16 en moyenne et basse montagne-citées dans ce travail-, et $\mathbf{4}$ autres à tendance orophile.

La plus grande richesse spécifique est observée dans deux localités de l'oued Choula ; à l'altitude de sa source (CO : $850 \mathrm{~m}$ ) et de son émissaire $(\mathrm{Cl}: 800$ $\mathrm{m}$ ), on recueil le respectivement 9 et 10 taxa (Tableau I) ; si nous prenons en compte les éléments non déterminés à l'espèce, nous arrivons à un total de 14 pour la station $\mathrm{Cl}$. D'une manière plus générale, une grande diversité ( 6 à 10 esp.) est observée la plupart du temps dans le crénon (SD, KO, SM, Z1, CO, $\mathrm{C} 1, \mathrm{SO}$ ) et dans un ruisseau côtier $(\mathrm{H})$ où, comptetenu de la faible longueur de l'oued (2 $\mathbf{~ k m}$ ), il s'agit aussi d'une station appartenant au crénal. Cette diversité importante du crénon de moyenne al titude ( $800-1000 \mathrm{~m})$ s'oppose à la pauvreté (1-3 esp.) du peuplement des cours d'eau de la vallée, à taux de salinìté et à température élevés. Dans certaines localités, cet te pauvreté est accentuée par la pollution $\left(\mathbf{B A}_{t}\right.$ MG, S2, S3: 1 seule espèce, Tableau I).

Dans 4 localités (T 10, Z2, HF et DJ), aucune Simulie n'a été récoltée ; la première station (T10) est un estuaire soumis à l'influence marine, les 3 autres sont caractérisées par une eau trouble, un courant très lent avec parfois une eau simplement renouvelée...

Dans la wilaya de Tlemcen, $S$. (W.) pseudequinum (36 st.) et $S$. (S.) intermedium (27 st.) sont dominantes par leur fréquence et leur abondance dans les divers biotopes ; le gr. aureum et $S$. (W.) sergenti sont fréquents ; S. (S.) gaudi (9 st.) et S. (S.) omatum (1 2 st.) 
Tableau IIl. - Répartition méditerranéenne des espèces recensées d'Algérie.

\begin{tabular}{|c|c|c|c|c|c|c|c|c|}
\hline $\begin{array}{l}\text { Taxa recensés } \\
\text { en Algérie }\end{array}$ & Maroc & $\begin{array}{l}\text { Algérie } \\
\text { (ouest) }\end{array}$ & Tunisie & $\begin{array}{l}\text { Péninsule } \\
\text { ibérique }\end{array}$ & $\begin{array}{l}\text { France } \\
\text { du sud }\end{array}$ & Italie & $\begin{array}{l}\text { Proche } \\
\text { Orient }\end{array}$ & Répartition \\
\hline G. fabri & + & + & & + & + & + & & Atlantique \& Médit. occ. \\
\hline P. (P.) faurei & + & + & & & & & & Endémique Afrique du Nord \\
\hline S. (E.) velutinum & + & + & + & + & + & + & +2 & Circumméditerranécennę \\
\hline S. (E.) angustipes & + & + & $?$ & + & + & + & + & Europe + Méditerranée \\
\hline S. (N.) costatum & + & + & & + & + & + & & Europe + Méditerranée \\
\hline S. (N.) ibleum & & + & + & & & + & + & Circumméditerranéenne \\
\hline S. (N.) latigonium & & + & & + & + & + & & Europe + Mediterranée \\
\hline S. (N.) ruficone & + & & + & + & & & & Afrique, Péninsule ibér. \\
\hline S. (Ob.) marocanum & + & + & & & & & & Endémique Afrique du Nord \\
\hline S. (S.) intermedium & + & + & + & + & $+^{*}$ & + & & Europe + Médit. occ. \\
\hline S. (S.) ornatum s. 1 & + & + & + & + & + & + & + & Europe + Méditerranée \\
\hline S. (S.) argenteostriatum & & & + & & + & + & & \\
\hline S. (S.) gaudi & + & + & & & + & & & Méditerranée occidentale \\
\hline S. (T.) bezzii & + & + & & + & + & + & + & Circumméditerranéenne \\
\hline S. (W.) pseudequinum & + & + & + & + & + & + & + & Circumméditerranéenne \\
\hline S. (W.) sergenti & + & + & + & + & & & & Méditerranée occidentale \\
\hline
\end{tabular}

peuvent être abondants dans certaines de leurs loca. lités. Les autres taxons sont faiblement représentés $-S$. (Ob.) marocanum, bien que présent dans une seule station, étant très abondant dans son biotope(Tableau II).

Par rapport à la Tunisie -où 6 espèces sont communes avec les nôtres-, l'élément dominant reste le même : $S$. (W.) pseudequinum. Par contre, $S$. (E.) velutinum, très fréquent et abondant en Tunisie, est remplacé dans notre région par $S$. (S.) intermedium.

Dans le Rif, au Nord-Ouest de la zone étudiée. Bailly-Choumara \& Beaucournu-Saguez (1978) ont recensé 12 espèces dont 8 sont communes avec les nôtres ; 4 n'ont pas été retrouvées pour l'instant: Metacnephia blanci, S. (N.) vernum, S. (N.) ruficorne et $S$. ( $S$.) variegatum.

Sur simple examen du tableau I, les Simulies peuvent être séparées en 3 groupes : 1) les espèces du crénal : $P$. (P) faurei P.(P.) sp., G. fabri, S. (Ob.) marocanum, S. (N.) ibleum, 2) les espèces de plaine (potamal) : $S$. (W.) sergenti ; 3 ) les espèces de plaine de la bordure septentrionale méditerranéenne que nous retrouvons ici remontant à toutes altitudes et dans divers biotopes, à cause de l'élévation de température de l'eau et de la salinité des cours d'eau de basse altitude : $S$. (S.) intermedium, $S$. (S.) omatum, S. (W.) pseudequinum... Cette remontée avait été notée par Giudicelli \& Dakki (1984) dans leur travail sur les sources du Maroc. Par ailleurs, elle se trouve déjà esquissée pour certaines espèces sur la limite occidentale du massif pyrénéen (Vinçon \& ClergueGazeau 1988). Les éléments de ce troisième groupe sont ainsi répandus le long des principaux cours d'eau depuis le crénal jusqu'au potamal. Les espèces du sous-genre Wilhelmia ont tendance à rester seules dans le potamal, à cause de leur tolérance à la salinité. Les éléments de basse montagne (hyporhithral) et de plaine (potamal) de la bordure nordpyrénéenne se retrouvent ici, dans le crénal de moyenne altitude, avec les espèces de sources. Il existe ainsi une opposition entre ce type de crénal à grande diversité spécifique et celui des hautes montagnes européennes à faible diversité, où les espèces, sténothermes d'eau froide, sont faiblement représentées.

L'étude biogéographique du peuplement confirme qu'il s'agit d'une faune paléarctique, répandue principalement dans la sous-région méditer ranéenne (Zwick 1978); seule, S. (W.) pseudequinum est d'orjgine orientale. Peuvent être distinguées (Tableau III) : les espèces de grandes plaines européennes qui descendent sur la bordure méditerranéenne, les espèces circumméditerranéennes, les espèces de la Méditerranée occidentale, les espèces at lantiques méditerranéennes et 4 espèces endémiques d'Afrique du Nord: $P$. (P.) faurei, $P$. (P.) sp., S. (N.) cf cryophilum et S. (Ob.) marocamum, dont on connait l'affinité pour certains éléments de la bordure nord-méditerranéenne. 
Si l'on compare ces premiers résultats avec ceux de l'étude hydrobiologique de l'oued Aïssi (Grande Kabylie), une différence importante s'observe chez les peuplements de Simulies de l"Est-algérien, avec la présence d'espèces nettement plus orophiles : $S$. (N.) cryophilum, S. (S.) variegatum, S. (S.) argenteostriatum, S. (S.) gr. monticola, $S$. (T.) bezzii, à cause d'un régime des eaux de type pluvio-nival et d'une forte vitesse de courant.

\section{Travaux cités}

Aït Mouloud (S.) 1987. Essai de recherches sur la dérive des macroinvertébrés aquatiques dans l'oued Alssi : faunistique, écologie et biogéographie. Thèse de Magister, Univ. Alger. $118 \mathrm{p}$.

Bailly-Choumara (H.), Bernard (M.R.), Grenier (P.), Le Roy-Moret (M.C.) \& Mouchet (J.). 1970. - Notes faunistiques sur les Simulies (Diptera: Simulidae) du nord de la Tunisic. Cah. O.R.S.T.M. série Ent. Méd. et Parasitol., 8 (4) : 377-382.

Bailly-Choumara (H.) \& Beaucourna-Saguez (F). 1978, Contribution à l'étude des Simulies du Maroc (Diptera : Simu liidae) 1) Le Rif. Bult. Inst. Scient. Rabat, (3) : 121-144.

Bailly-Choumara (H.) \& Beaucournu-Saguez (F.) 1981. Cont ribution à l'étude des Simulies (Diptera : Simulidae) du Maroc. 2) le Haut-Atlas. Bull Inst. Scient. Rabat., (5); $39-57$.

Beaucournu-Saguez (F.), Skierska (B.) \& Rault (B.). 1969. Observations sur le peuplement en stades immatures de Sirnu liidae (Diptera : Nematocera) en Bretagne, dans cinq gîtes sujvis pendant un cycle annuel. Bull. Soc. Sci. Bretagne, 44 209.229 .

Beaucournt-Saguer (F.) 1972. - Captures de Simulies dars la moitie sud du Portugal. Premières captures en Eu rope de Simutlium sergenti Edwards 1923 et de Simulium ruficome Macquart 1838. Ar. Esc. nac. Saude publi. e de Med. trop., $6(1-4)$ : 73-83.

Beaucournu-Saguez (F.). 1975. - Récoltes de Simulies (Diptera : Simuliidae) dans le Sud-Est de l'Espagne. Annls Soc, entomol. Fr. (N.S.). 11(1): 73-79.

Beaucournu-Saguez (F.) \& Braverman (Y.). 1987. - Greniera dobyi (Diptera, Simuliidae) espèce nouvelle du Golan (Israël). Annls Parasicol. Hum comp., 62, (1): 77-91.

Bernard (M.R.), Grenier (P.) \& Bailly-Choumara (H.). 1972. Description de Prosimudium (Prosimudium) faurei n. sp., (Diptera : Simuliidae), Cah. O.R.S.T.O.M. série Ent. méd. el Parasitol., $10(1): 63-68$.

Boumaiza (M.) \& Clergue-Gazeau (M.) 1986. - Le peuplement simulidien de la Tunisie. I. Inventaire faunistique et biogéographie. (Diptera : Nematocera). Annls Limmol., 22 (1): 31-39.
Bouzidi (A.) \& Giudicelli (I.). 1987. - Contribution à l'étude fau nistique et écologique des Simulies du Maroc (Diptera, Simu liidae). II. Simulium (Obuchovia) marocanum n. sp. et les espè. ces méditerranéennes d'Obuchovia Rubzov. Annls Limnol., 23 : (3) : 185-195.

Crosskey (R.W.). I981. - Simuliid taxonomy. The contemporary Scene 3-18. In : Blackflies, the future for biological methods in integrated control; Laird (M.) ed. Academic Press. London : $399 \mathrm{p}$.

Crosskey (R.W.). 1987. - An annotated checklist of the world blackflies (Diptera : Simuliadae) : 425-520. In : Black flies. Eco logy, population management, and annotated world list. Kin (K.) \& Merrit (R.W.) Ed. Pennsylvania State University Press London.

Doby (J.M.) \& David (F.J. 1959, - Description de Greniera fabri Doby \& David, 1959 (Diptera-Nematocera-Simuliidae) en provenance de l'Ouest de la France (Loire-Atlantique). Bull. Soc Pathol exot., 52 (5) : 669.678.

Edward (F.W.). 1923. - On some algerian species of Simulitium Arch. Inst. Pasteur. Algérie., 1 (4) : 647-653.

Gauthier (H). 1928. - Recherches sur la faune des eaux continen tales de i'Algérie et de la Tunisie. Minerva, Alger; 149 p.

Giudicelli (J.) \& Dakki (M.). 1984. - Les sources du Moyen Allas et du Rif (Maroc) : faunistique (description de deux especes nouvelles de Trichoptères), écologié, intérêt biogéographique. Bij dragen tol de Dierkitide. 54 (1):83-100.

Gonzàlez-Peña (G.), Ferreras Romeno (M.) \& Garcia Rojas (A.) 1986. - Introduction al estudio de los simulidos (Diptera) de Sierra Morena (Sur de España) : Rio Yeguas. Acta de las VJIJ Jomadas $A$ e E, Sevilla. 733-744.

Grenier (P.). 1953. - Simuliidae de France et d'Afrique du Nord. (Systématique, biologie, importance médicale). Encyclop. Ento mol., Série A $29: 1.170$.

Grenier (P.) \& Faure (R.). 1956. - Description d'une Simulie nouvelle du Maroc Simulitem gaudi n. sp. (Diptera, Simuliidael Bull. Soc. Path. exolique, 49 (5): 838-840.

Gremier (P.), Faure (P.R.) \& Laurent (J.). 1957. - Simulies (Diptera Simuliidae) du Maroc (deuxième mémoire) Arch. Inst. Pasteur Maroc., 5 (6) : 218-242.

Lounaci (A.). 1987. - Recherches hydrobiologiques sur les peuplements d'Invertebrés benthiques du bassin de l'oued Aiss (gde Kabylie). Thè se Magister (Eco-Ethologie). Alger. 133 p.

Parrot (L.). 1949. - Quelques notes sur les Simulies d'Algérie Arch. Inst. Passeur, Algérie, 27 (3) : 273-275.

Rivosecchi (L.\}. 1978. - Simulitala Diptera Nematocera. Fauna Italia, Academia Nazionale Italia di Entomol. e del Unione Zool. Italiana Bologna. 13 VHI : $533 \mathrm{p}$.

Vaillant (F.). 1955. - Recherches sur la faune madicole de France de Corse et d'Afrique du Nord. Mem. Mus. Hist. nut. Pari. (Zool.)., I I : 1-258 + VI Pl.

Vinçon (G.) \& Clergue-Gazeau (M.). 1988. - Etude hydrobiologique de la vallée d'Ossau (Pyrénées Atlantiques, France). III Simuliidae (Diptera, Nematocera) : leur originalité biogéogra. phique et écologique. Annts Limnol., 24 (1): 67-81.

Zwick (H.). 1978. - Simuliidae (Diptera). In : Limno Jauna Euro paea. Illies (J.) Ed : 396-403. 